\title{
COMPUTATIONAL AEROACOUSTICS OF VISCOUS LOW SPEED FLOWS USING SUBGRID SCALE FINITE ELEMENT METHODS
}

\author{
ORIOL GUASCH \\ GTAM - Grup de Recerca en Tecnologies Audiovisuals i Multimedia \\ Enginyeria i Arquitectura La Salle, Universitat Ramon Llull, \\ C/ Quatre Camins 2, Barcelona 08022, Catalonia (Spain) \\ oguasch@salle.url.edu \\ http://www.salle.url.edu/ oguasch \\ RAMON CODINA \\ UPC, Universitat Politècnica de Catalunya \\ C/ Jordi Girona 1-3, Edifici C1, Barcelona 08034, Catalonia (Spain) \\ ramon.codina@upc.edu \\ http://www.rmee.upc.es/homes/codina \\ Received (Day Month Year) \\ Revised (Day Month Year)
}

\begin{abstract}
A methodology to perform computational aeroacoustics of viscous low speed flows in the framework of stabilized finite element methods is presented. A three-step procedure is followed that makes use of Lighthill's acoustic analogy. In the first step, the incompressible Navier-Stokes equations are solved to obtain the flow velocity field. In the second step, the acoustic source term is computed from this velocity field and then Fourier transformed to the frequency domain. Finally, the acoustic pressure field is obtained by solving the Fourier transform of Lighthill's acoustic analogy. All equations in the formulation are solved using subgrid scale stabilized finite element methods. The main ideas of the subgrid scale numerical strategy are outlined and its benefits when compared to the Galerkin approach are described. As numerical examples, the aerodynamic noise generated by flow past a two dimensional cylinder and by flow past two cylinders in parallel arrangement are addressed.
\end{abstract}

Keywords: Computational aeroacoustics; Lighthill's analogy; variational multiscale; residual based stabilization; subgrid scale finite element.

\section{Introduction}

Aeroacoustics is concerned with noise generated by unsteady and/or turbulent flows and also by their interaction with solid boundaries. This type of noise is commonly known as aerodynamic noise. In contrast to classical acoustics, forces and motions inside the flow are the sources of noise rather than the externally applied ones. On the other hand, computational aeroacoustics (CAA) is a relatively recent computational field aiming at the simulation and prediction of aerodynamically generated noise. In this paper, a methodology is presented to compute aerodynamic noise generated by viscous subsonic flows in the framework of stabilized finite element methods. The herein proposed strategy was used in a previous work by the authors ${ }^{1}$ to illustrate the performance of a stabilized finite element 
method for the convected Helmholtz equation, when applied to aeroacoustics. However, the CAA strategy was only briefly commented there, so in this paper we aim at complementing our previous work by exposing the CAA approach in full detail.

Central to aeroacoustics is the pioneering work of Lighthill who introduced the concept of acoustic analogy. Lighthill ${ }^{2}$ established an analogy between the compressible Navier-Stokes equations and the sound radiated by a distribution of quadrupole sources in a quiescent media, for the case of unbounded flows. The analogy has the expression

$$
\left(\partial_{t t}^{2}-c_{0}^{2} \nabla^{2}\right)\left(\rho-\rho_{0}\right)=(\nabla \otimes \nabla): \mathbf{T},
$$

where $\rho^{\prime}:=\rho-\rho_{0}$ denotes the density fluctuations, $c_{0}$ is the sound speed, $\partial_{t}$ stands for the partial time derivative, $\otimes$ is the tensor product and $T$ is the so called Lighthill tensor given by

$$
\mathbf{T}:=\rho(\boldsymbol{u} \otimes \boldsymbol{u})+\left[\left(p-p_{0}\right)-c_{0}^{2}\left(p-p_{0}\right)\right] \mathbf{I}-\sigma .
$$

In Eq. (2), $u$ represents the velocity vector, $p^{\prime}:=p-p_{0}$ is the pressure fluctuation, $\mathbf{I}$ is the identity tensor and $\sigma$ is the Cauchy stress tensor. Traditionally, Eq. (1) has been solved assuming a known value for $\mathrm{T}$ (actually for an approximation to it) and convolving it with

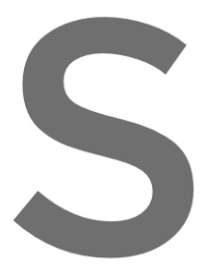
the acoustic free sp To account for function, other analo dipolar character in the obtained by $\mathrm{Curle}^{3}$ for the case of non-movin
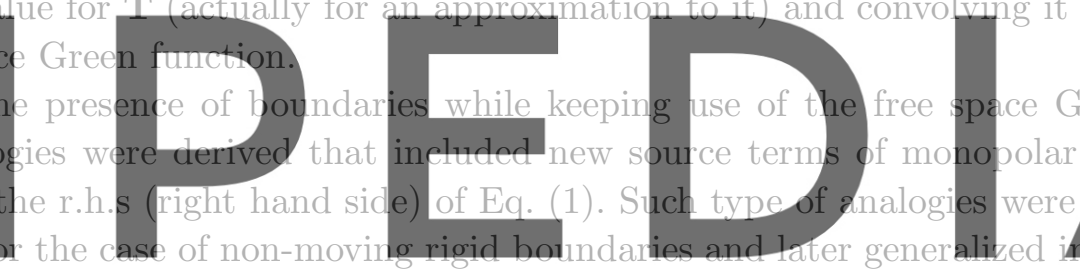

well-known Ffowcs-Williams Hawkings equation ${ }^{4,5,6}$ ). On the other hand, further analogies

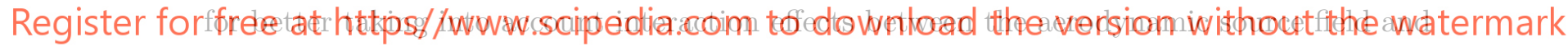
the acoustic one can be derived. Some well-known analogies are those of Lilley, Phillips, Ribner and Legendre (see e.g., Ref. 7 for a review). Analogies that emphasize the role of vorticity in the aerodynamic noise generation process have been also developed ${ }^{8,9,10}$ and it should be mentioned that the development of new acoustic analogies is still a subject of current research ${ }^{11}$.

In this paper, however, we will concentrate on solving the time Fourier transform of the original Lighthill acoustic analogy, given by Eq. (1), and we will make use of the Reynolds tensor to approximate Lighthill's tensor. That is, in the case of high Reynolds number problems with uniform mean density and low Mach number, Lighthill's tensor can be replaced by the Reynolds tensor

$$
\mathbf{T} \approx \rho_{0}(\boldsymbol{u} \otimes \boldsymbol{u})
$$

where $\boldsymbol{u}$ stands now for the velocity vector of the flow that is assumed to be incompressible, i.e. $\nabla \cdot \boldsymbol{u}=0$. This approximation, already proposed by Lighthill, is by no means evident and its range of validity was justified by $\mathrm{Crow}^{12}$ using the method of Matched Asymptotic Expansions. More recently, Ristorcelli ${ }^{13}$ performed a two-time perturbation analysis of the problem and proposed a compressibility correction to the Reynold's tensor only involving the solenoidal flow velocity and pressure (see also the related work in Ref. 14). 
In order to solve the inhomogeneous Helmholtz equation that results from the time Fourier transform of Eq. (1), together with the approximation in Eq. (3), a methodology is proposed that can be conceptually divided in three steps. In the first step a computational fluid dynamic (CFD) simulation of the incompressible Navier-Stokes is performed to obtain the flow velocity field. In the second step the source term in the r.h.s of Eq. (1) i.e., the double divergence of the Reynolds tensor, is computed and time Fourier transformed. Finally, in the third step the result is inserted in the inhomogeneous Helmholtz equation corresponding to the time Fourier transform of Eq. (1) and solved to obtain the acoustic pressure field. This procedure avoids some of the problems found when using the standard Green functions approach such as evaluating the source term at appropriate retarded times. It also accounts for the presence of boundaries in a natural way and allows a direct visualization of the acoustic source term.

The differential equations in the first and third steps of the method have been solved using subgrid scale stabilized finite element methods. For the incompressible Navier-Stokes equations, a stabilization method recently developed by the second author and co-workers has been implemented ${ }^{15,16,17,18}$. The method accounts for the time variation of the subgrid scales and may be a possible numerical alternative to LES (Large Eddy Simulation)

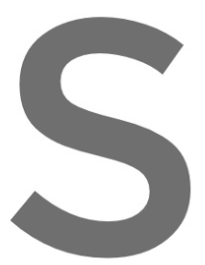
approaches for turbulent flo braic subgrid scale the Galerkin Least-Sq Helmholtz equation that in Refs. 24 and
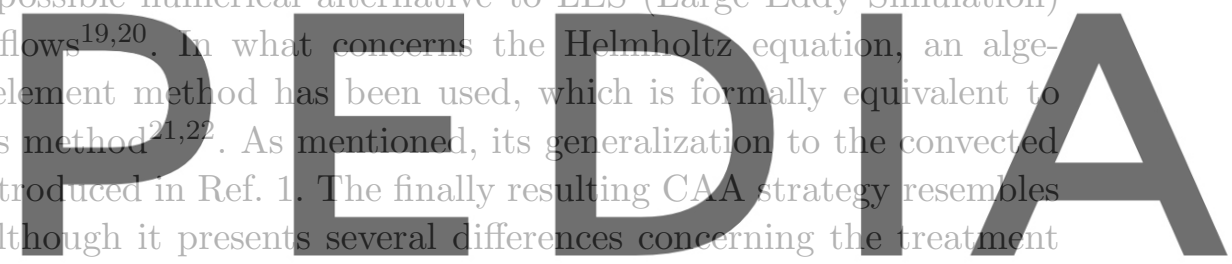

of the acoustic source term, the stabilized weak forms used in the numerical formulation,

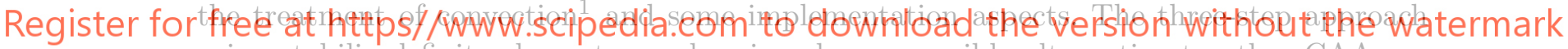
using stabilized finite elements can be viewed as a possible alternative to other CAA approaches such as the use of acoustic analogies involving the integral formulation of the Ffowcs-Williams and Hawkings equation ${ }^{28,26,27}$, the use of dispersion-relation-preserving schemes $^{29}$ and its grid-optimized version ${ }^{30}$, the use of hybrid approaches with high order schemes ${ }^{31}$ or the space-time and solution-element approach in the framework of finite volumes ${ }^{32}$, among others.

The paper is organized as follows. In section 2 the proposed methodology is formulated in detail. In section 3 the weak forms corresponding to the differential equations to be solved are given. The Galerkin finite element approach to solve these weak forms is presented and its problems outlined. The stabilized finite element methods that avoid the typical problems from the Galerkin approach problems are formulated in section 4 . In section 5, two numerical examples dealing with aerodynamic noise generated by flow past single and parallel cylinders for different Reynolds numbers are presented. Conclusions are finally drawn in section 6 . 


\section{Problem Statement}

\subsection{First step: Computational Fluid Dynamic Simulation}

The CFD computation aims at obtaining the flow velocity vector, $\boldsymbol{u}$, from the solution of the time evolving incompressible Navier-Stokes equations. The mathematical problem consists in solving the latter equations in a given computational domain $\Omega \subset \mathbb{R}^{d}$ (where $d=2$ or 3 is the number of space dimensions) with boundary $\partial \Omega$ and prescribed initial and boundary conditions. Splitting $\partial \Omega$ into two disjoint sets $\partial \Omega=\overline{\Gamma_{D} \cup \Gamma_{N}}$ respectively accounting for those boundaries with prescribed Dirichlet and Neumann conditions, the problem to be solved reads

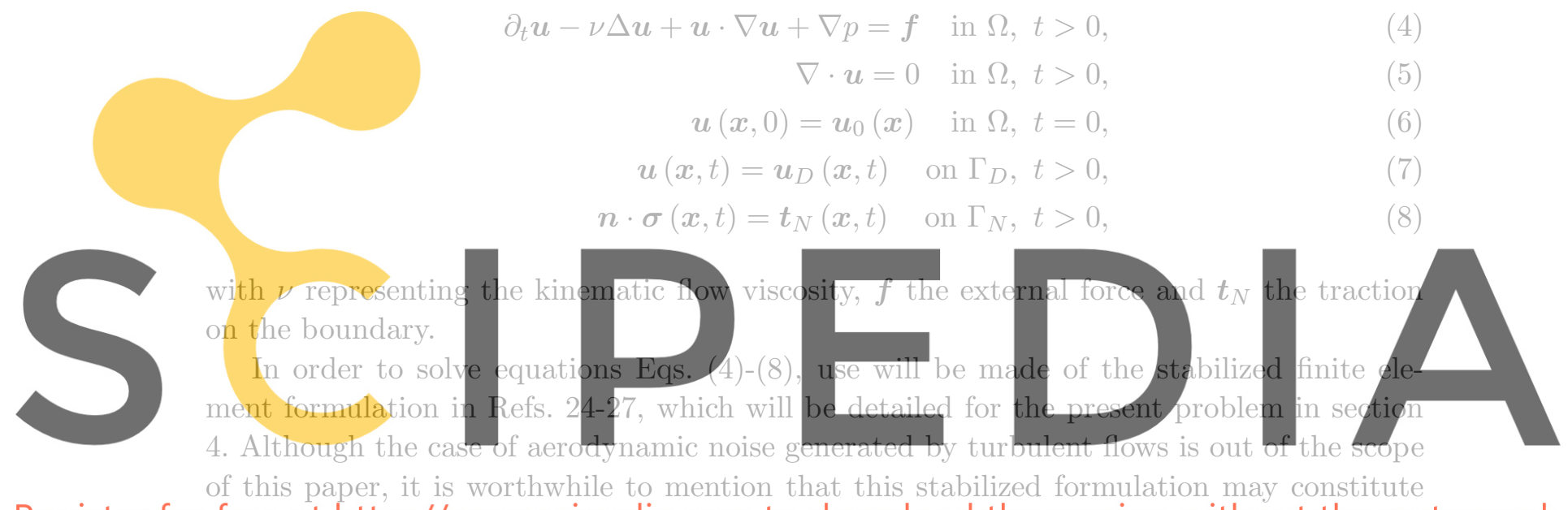

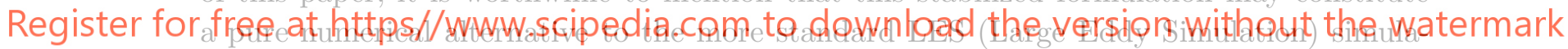

tion for high Reynolds number flows ${ }^{17}$. It is to be noted that while LES performs a scale

decomposition for the velocity and the pressure fields by convolving Eqs. (4)-(8) with a filter function ${ }^{33,34}$, the scale decomposition is performed in the stabilization framework by means of a projection onto the finite element space ${ }^{35,36,37}$. The large scales represent those scales that can be directly captured with the computational mesh, whereas the small scales represent those scales that cannot be captured by the mesh. The idea that the extra terms appearing in the equations as a result of this separation could suffice to simulate turbulent flows, seems to be supported by several numerical examples involving the decay of isotropic turbulence and the development of turbulence in channel flows $38,39,40,41,19,20$.

\subsection{Second step: The source term}

The second step of the method consists in obtaining the acoustic source term, i.e., $\rho_{0}(\nabla \otimes \nabla):(\boldsymbol{u} \otimes \boldsymbol{u})$, from the flow velocity vector, $\boldsymbol{u}$, computed in the first step of the method. As the source term involves a double divergence it cannot be directly computed using finite elements of class $C^{0}$, unless it is integrated by parts in the weak form of the problem transferring one derivative to the test function ${ }^{24}$. However, there exists also the possibility to approximate the source term with first order derivative terms thanks to the 
incompressibility constraint. Effectively,

$$
\begin{aligned}
(\nabla \otimes \nabla): \mathbf{T} & \approx \rho_{0}(\nabla \otimes \nabla):(\boldsymbol{u} \otimes \boldsymbol{u}) \\
& \approx \rho_{0}(\nabla \otimes \boldsymbol{u}):(\nabla \otimes \boldsymbol{u})^{T}=: s(\boldsymbol{x}, t),
\end{aligned}
$$

where $\nabla \cdot \boldsymbol{u}=0$ has been used twice and we have defined $s(\boldsymbol{x}, t)$ in the last line. This approximation allows the direct visualization of the source term while keeping the advantages of using $C^{0}$-class finite elements.

The second step of the method finishes by performing the time Fourier transform of $s(\boldsymbol{x}, t)$ to get $\hat{s}(\boldsymbol{x}, \omega)$. An important implementation aspect is that of avoiding the storage of $s(x, t)$ to compute its transform. This can be done by choosing a priori the frequency range of interest and, during the CFD evolution, computing $s(x, t)$ for each time step as well as its contribution to $\hat{s}(\boldsymbol{x}, \omega)$. At the end of the computation only the latter is stored. Hence, although steps I and II of the methodology have been differentiated for the sake of clarity, in practice they are carried out simultaneously.

\subsection{Third step: Computing the acoustic field}
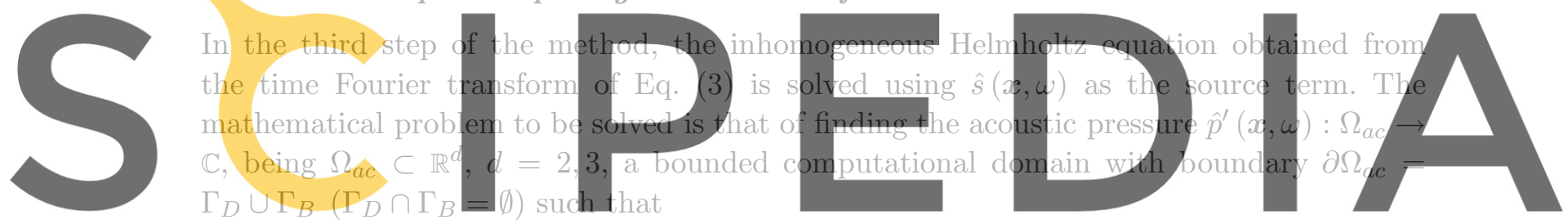

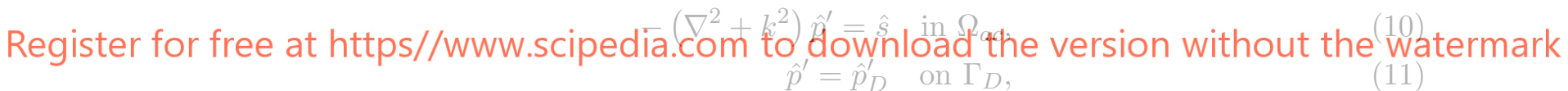

$$
\nabla \hat{p}^{\prime} \cdot n=M\left[\hat{p}^{\prime}\right]+\hat{g} \quad \text { on } \Gamma_{B} \text {. }
$$

In Eqs. (10)-(12) $k=\omega / c_{0}$ is the wavenumber, $\boldsymbol{n}$ stands for the normal pointing outwards $\Gamma_{B}, \hat{g}: \Gamma_{B} \rightarrow \mathbb{C}$ represents prescribed data on $\Gamma_{B}$ and $M\left[\hat{p}^{\prime}\right]$ is an integral operator. For exterior problems, $M\left[\hat{p}^{\prime}\right]$ defines a non-reflecting condition and $\Gamma_{B}$ the external boundary. If $\Gamma_{B}$ is far enough from the source region, the non-local boundary condition specified by $M\left[\hat{p}^{\prime}\right]$ can be replaced by the local condition $\mathrm{i} k \hat{p}^{\prime}$. Eq. (12) then becomes a Robin type boundary condition (Sommerfeld's radiation condition),

$$
\nabla \hat{p}^{\prime} \cdot \boldsymbol{n}=\mathrm{i} k \hat{p}^{\prime}+\hat{g} \quad \text { on } \Gamma_{B}
$$

\section{Galerkin finite element approximation}

\subsection{Spatial continuous weak forms}

\subsubsection{Time-discrete spatial-continuous weak form of the Navier-Stokes equation}

To find a numerical solution to Eqs. (4)-(8) we have to discretize them in time and space.

The time discretization has been carried out using the generalized trapezoidal rule. Actually, 
the second order Crank-Nicolson scheme has been used in the computations. Let us consider a partition of the computational time interval $0<t^{0}<\ldots<t^{N}$, a constant time step size $\delta t:=t^{n+1}-t^{n}$, and let us introduce the following notation for a generic time-dependent function $h(t)$,

$$
\begin{gathered}
\delta h^{n}:=h^{n+1}-h^{n}, \\
h^{n+1 / 2}:=\frac{1}{2}\left(h^{n+1}+h^{n}\right), \delta_{t} h^{n}:=\delta h^{n} / \delta t,
\end{gathered}
$$

where $h^{n}$ stands for the value of $h$ at time $t^{n}$. The time discrete version of the Navier-Stokes Eqs. (4)-(8) can then be written as

$$
\begin{array}{rc}
-1 / 2+\nabla p^{n+1}=f^{n+1 / 2} & \text { in } \Omega, \\
\nabla \cdot u^{n+1 / 2}=0 & \text { in } \Omega, \\
u^{0}=u_{0} & \text { in } \Omega, \\
u^{n+1 / 2}=u_{D}^{n+1 / 2} & \text { on } \Gamma_{D}, \\
n \cdot \sigma^{n+1 / 2}=t_{N}^{n+1 / 2} & \text { on } \Gamma_{N} .
\end{array}
$$

We next have t solve these equations using
variational continuous form.
\[ \mathcal{V}_{0}^{d} \equiv \boldsymbol{H}_{0}^{1}(\Omega): \]

$$
\mathcal{Q}_{0}:=\{
$$

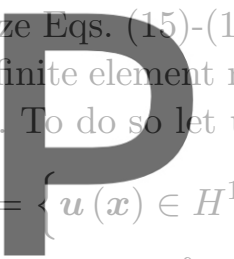<smiles></smiles>

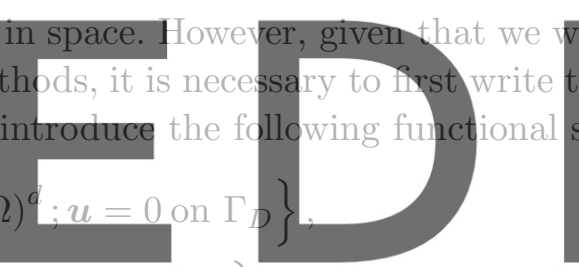

where $L^{2}(\Omega)$ denotes the space of square integrable functions (real or complex) in the domain $\Omega$, and $H^{1}(\Omega)$ is the first order Sobolev space of functions with integrable first derivatives. Let us also consider in what follows, and for simplicity of exposition, the case of homogeneous Dirichlet conditions (the extension to the inhomogeneous can be done in the usual manner). The weak or variational form corresponding to Eqs. (15)-(19) with $\boldsymbol{u}_{D}^{n}=0$ is found multiplying these equations by test functions $\boldsymbol{v} \in \mathcal{V}_{0}^{d}, q \in \mathcal{Q}_{0}$ and integrating over the whole domain $\Omega$. The variational problem can be then formulated as: from known $\boldsymbol{u}^{n} \in \mathcal{V}_{0}^{d}$, find $\boldsymbol{u}^{n+1 / 2} \in \mathcal{V}_{0}^{d}, p^{n+1} \in \mathcal{Q}_{0}$ such that

$$
\begin{aligned}
& \int_{\Omega} \boldsymbol{v} \cdot\left[\delta_{t} \boldsymbol{u}^{n}+\left(\boldsymbol{u}^{n+1 / 2} \cdot \nabla \boldsymbol{u}^{n+1 / 2}\right)\right] d \Omega+\nu \int_{\Omega} \nabla \boldsymbol{v}: \nabla \boldsymbol{u}^{n+1 / 2} d \Omega \\
& -\int_{\Omega} p^{n+1} \nabla \cdot \boldsymbol{v} d \Omega=\int_{\Omega} \boldsymbol{v} \cdot \boldsymbol{f}^{n+1 / 2} d \Omega+\int_{\Omega} \boldsymbol{v} \cdot \boldsymbol{t}_{N}^{n+1 / 2} d \Gamma_{N} \\
& \int_{\Omega} q \nabla \cdot \boldsymbol{u}^{n+1 / 2} d \Omega=0
\end{aligned}
$$

for all $\boldsymbol{v} \in \mathcal{V}_{0}^{d}, q \in \mathcal{Q}_{0}$.

In order to shorten the notation in Eqs. (21)-(22) and subsequent equations, we will use the brackets, $\langle\cdot, \cdot\rangle$ to denote the integral of the product of any pair of distributions $f$ and $g$ 
in the domain $\Omega$, i.e.,

$$
\langle f, g\rangle \equiv \int_{\Omega} f g d \Omega
$$

In particular, $\langle\cdot, \cdot\rangle$ can represent the duality pairing in $\mathcal{V}_{0}^{d}$ and for $f, g \in L^{2}(\Omega)$ it will correspond to the inner product in this space, which will be designated by $(\cdot, \cdot)$. For the surface integrals use will be made of $\langle\cdot, \cdot\rangle_{\Gamma}$ and $(\cdot, \cdot)_{\Gamma}$ with analogous meanings. We also introduce the following notation

$$
l(\boldsymbol{v}):=\langle\boldsymbol{v}, \boldsymbol{f}\rangle+\left\langle\boldsymbol{v}, \boldsymbol{t}_{N}\right\rangle_{\Gamma_{N}} .
$$

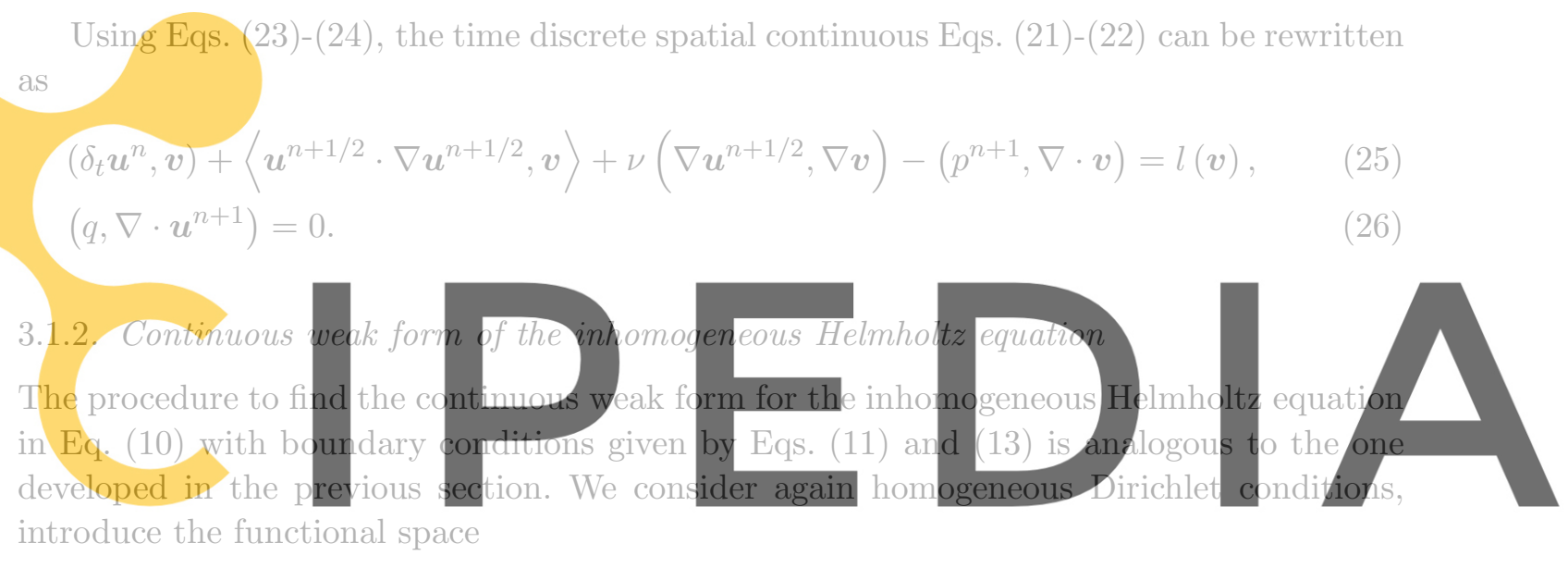

\section{Register for free at https//www.scipedia.com to download the version without the watermark}

and muitiply Eq. (10) by a test function $w \in \mathcal{V} \mathcal{V}_{0}$. Finally, we integrate over the acoustic domain $\Omega_{a c}$. Using the notation in Eq. (23), the weak problem can be formulated as: find $\hat{p}^{\prime} \in \mathcal{W}_{0}$ such that

$$
\left(\nabla \hat{p}^{\prime}, \nabla w\right)-k^{2}\left(\hat{p}^{\prime}, w\right)-\mathrm{i} k\left(\hat{p}^{\prime}, w\right)_{\Gamma_{B}}=\langle\hat{s}, w\rangle+\langle\hat{g}, w\rangle_{\Gamma_{B}}
$$

for all $w \in \mathcal{W}_{0}$. Note that the acoustic pressure is a complex number so the scalar products in Eq. (28) involve the complex conjugate of the test function. Notice also that domain integrations in the scalar products take place now in $\Omega_{a c}$.

\subsection{Galerkin discrete weak forms}

\subsubsection{Galerkin finite element approximation of the Navier-Stokes equation}

Let us now proceed to the spatial discretization of Eqs. (25)-(26). Given a finite element partition of $\Omega$ with $n_{\mathrm{el}}$ elements, $n_{\mathrm{u}}$ nodes for the velocity, $n_{\mathrm{p}}$ nodes for the pressure and the finite dimensional subspaces $\mathcal{V}_{0, h}^{d} \subset \mathcal{V}_{0}^{d}$, and $\mathcal{Q}_{h, 0} \subset \mathcal{Q}_{0}$ to respectively approximate the velocity and the pressure, the Galerkin finite element approach to Eqs. (25)-(26) can be 
stated as: from known $\boldsymbol{u}_{h}^{n} \in \mathcal{V}_{0, h}^{d}$, find $\boldsymbol{u}_{h}^{n+1 / 2} \in \mathcal{V}_{0, h}^{d}, p_{h}^{n+1} \in \mathcal{Q}_{h, 0}$ such that

$$
\begin{aligned}
& \left(\delta_{t} \boldsymbol{u}_{h}^{n}, \boldsymbol{v}_{h}\right)+\nu\left(\nabla \boldsymbol{u}_{h}^{n+1 / 2}, \nabla \boldsymbol{v}_{h}\right)+\left\langle\boldsymbol{u}_{h}^{n+1 / 2} \cdot \nabla \boldsymbol{u}_{h}^{n+1 / 2}, \boldsymbol{v}_{h}\right\rangle \\
& -\left(p_{h}^{n+1}, \nabla \cdot \boldsymbol{v}_{h}\right)+\left(q_{h}, \nabla \cdot \boldsymbol{u}_{h}^{n+1}\right)=l\left(\boldsymbol{v}_{h}\right)
\end{aligned}
$$

for all $\boldsymbol{v}_{h} \in \mathcal{V}_{0, h}^{d}, q_{h} \in \mathcal{Q}_{h, 0}$. The domain integrals in Eq. (29) are to be understood as $\int_{\Omega}:=\sum_{n_{\mathrm{el}}} \int_{\Omega_{\mathrm{el}}}$, with $\Omega_{\mathrm{el}}$ denoting an element domain, and $\boldsymbol{f}, \boldsymbol{t}_{N}$ in $l\left(\boldsymbol{v}_{h}\right)$ (r.h.s of Eq. (29)) are assumed continuous and evaluated at the time step $n+1 / 2$.

On the other hand, $\boldsymbol{u}_{h}$ and $p_{h}$ are of the type

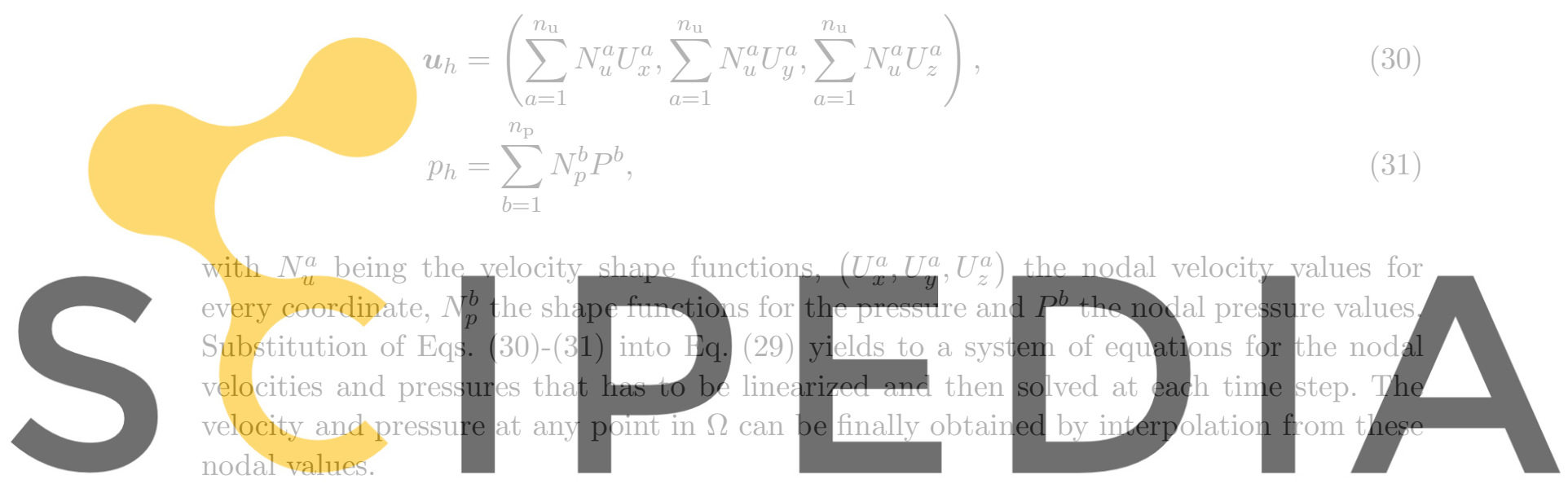

It is well known that the Galerkin formulation, Eq. (29), suffers from several numerical

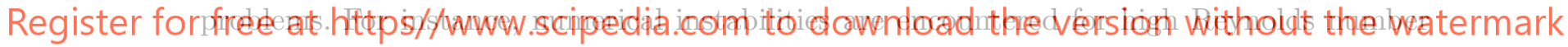
problems i.e., when the non-linear convective term in the equation dominates the viscous one. Moreover, a compatibility condition (inf-sup or LBB condition) is required to control the pressure term. This condition does not allow to use equal order interpolations to approximate the velocity and the pressure. This is certainly a problem because the use of equal order polynomials results in a much easier implementation of the numerical method as well as to the saving of computational time. On the other hand, further numerical instabilities are found when small time steps are used, specially at early stages of evolutionary processes. To circumvent all these difficulties that turn the Galerkin formulation Eq. (29) useless in practice, a subgrid scale stabilized formulation is presented in section 4 .

\subsubsection{Galerkin finite element approximation of the inhomogeneous Helmholtz equation}

The discrete weak form corresponding to the Galerkin finite element approximation of Eq. (28) can be stated as follows: given a finite element partition of $\Omega_{a c}$ with $n_{\text {ael }}$ elements and $n_{\text {ap }}$ nodes, and the finite dimensional subspaces $\mathcal{W}_{0, h} \subset \mathcal{W}_{0}$, find $\hat{p}_{h}^{\prime} \in \mathcal{W}_{0, h}$ such that

$$
\left(\nabla \hat{p}_{h}^{\prime}, \nabla w_{h}\right)-k^{2}\left(\hat{p}_{h}^{\prime}, w_{h}\right)-\mathrm{i} k\left(\hat{p}_{h}^{\prime}, w_{h}\right)_{\Gamma_{B}}=\left\langle\hat{s}_{h}, w_{h}\right\rangle+\left\langle\hat{g}, w_{h}\right\rangle_{\Gamma_{B}}
$$


for all $w_{h} \in \mathcal{W}_{0, h}$. Again,

$$
\hat{p}_{h}^{\prime}=\sum_{a=1}^{n_{\mathrm{ap}}} N_{p}^{a} \hat{P}^{\prime a},
$$

with $N_{p}^{a}$ being the acoustic pressure shape functions (that will be taken equal to those in Eq. (31) for this work) and $\hat{P}^{\prime a}$ the acoustic pressure nodal values.

The Galerkin weak form in Eq. (32) also presents numerical difficulties. The weak form becomes non-positive definite for large wavenumbers and it can be shown that the problem inf-sup constant presents an inverse dependence ${ }^{42}$ with the wavenumber $k$. This leads to a loss of stability and to the appearance of the so-called pollution error for large values of $k$. A dispersion analysis shows that this error is related to the fact that discrete waves propagate with a discrete wavenumber $k_{h} \neq k$. The difference $k_{h}-k$ increases for large wavenumbers and a phase error appears in the numerically computed waves. A subgrid scale stabilized finite element formulation will be also used in section 4 to avoid these difficulties.

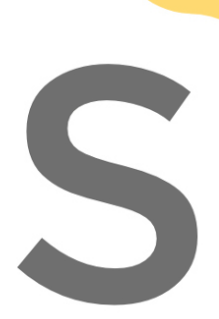

4. Stabilized finite element methods
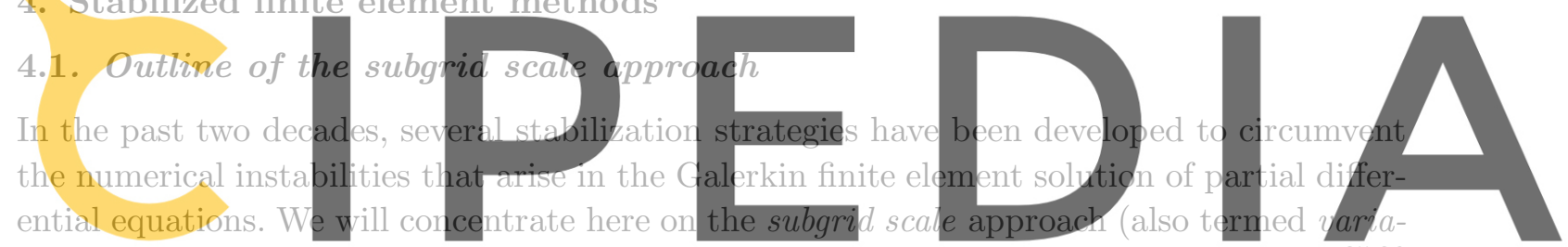

tional multiscale method or residual-based stabilization) originally developed by Hughes ${ }^{35,36}$

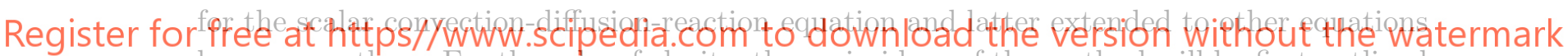

by many authors. For the sake of clarity, the main ideas of the method will be first outlined

for an abstract variational problem and then explicitly presented in detail for Eqs. (25)-(26) and Eq. (28) in subsequent sections.

Let us consider the abstract variational continuous problem of finding $y \in \mathcal{Y}$ such that

$$
m(y, z)=n(z)
$$

for all $z \in \mathcal{Z}$. $m$ and $n$ respectively represent (for simplicity) bilinear and linear continuous weak forms, while $\mathcal{Y}$ and $\mathcal{Z}$ are infinite dimensional spaces. The subgrid scale approach to find a numerical solution to Eq. (34) consists in first splitting $\mathcal{Y}$ and $\mathcal{Z}$ into $\mathcal{Y}=\mathcal{Y}_{h} \oplus \tilde{\mathcal{Y}}$ and $\mathcal{Z}=\mathcal{Z}_{h} \oplus \tilde{\mathcal{Z}} . \mathcal{Y}_{h}$ and $\mathcal{Z}_{h}$ represent the finite dimensional spaces (discrete spaces) where the numerical solution belongs, while $\tilde{\mathcal{Y}}$ and $\tilde{\mathcal{Z}}$ represent infinite dimensional spaces (continuous spaces) to respectively complete $\mathcal{Y}_{h}, \mathcal{Z}_{h}$ in $\mathcal{Y}$ and $\mathcal{Z}$. Variables $y$ and $z$ can then be decomposed as $y=y_{h}+\tilde{y}, z=z_{h}+\tilde{z}$ and substituted in Eq. (34) to obtain

$$
\begin{aligned}
m\left(y_{h}, z_{h}\right)+m\left(\tilde{y}, z_{h}\right) & =n\left(z_{h}\right), \\
m\left(y_{h}, \tilde{z}\right)+m(\tilde{y}, \tilde{z}) & =n(\tilde{z}) .
\end{aligned}
$$

Consequently, Eq. (34) has been transformed in two equations, Eq. (35) governing the dynamics of the resolvable "large" scales and Eq. (36) governing the dynamics of the "small" 
subgrid scales. The key idea consists in finding an approximate solution or model for the subscales equation, substitute it in the large scales equation and solve for them. In other words, the subgrid scale approach aims at simulating the influence of those small scales of the continuous problem, which cannot be captured by the numerical discretization, onto the numerical solution. The influence of these small continuous scales is what is not taken into account in the Galerkin numerical approach to the problem.

Note that the separation between scales performed in the subgrid scale approach Eqs. (35)-(36) is based on a projection onto the spaces $\mathcal{Z}_{h}$ and $\tilde{\mathcal{Z}}$, and that the modeling for the subscales is carried out once the problem has been already discretized. As mentioned in section 2.1, this is to be compared with the filtering approach to LES where the scale separation and modeling are performed at the continuum level, prior to any discretization.

\subsection{Subgrid scale stabilized finite element method for the Navier-Stokes equations}

To apply the subgrid scale finite element method to Eqs. (25)-(26) we will decompose the velocity and velocity test function as $u^{n}=u_{h}^{n}+\tilde{u}^{n}, u^{n+1 / 2}=u_{h}^{n+1 / 2}+\tilde{u}^{n+1 / 2}$ and
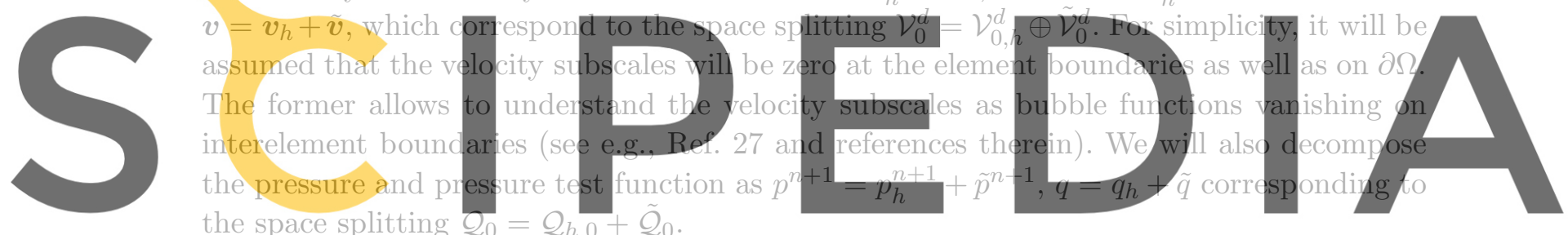
the space splitting $\mathscr{Q}_{0}=\mathcal{Q}_{h, 0}+\tilde{\mathcal{Q}}_{0}$.

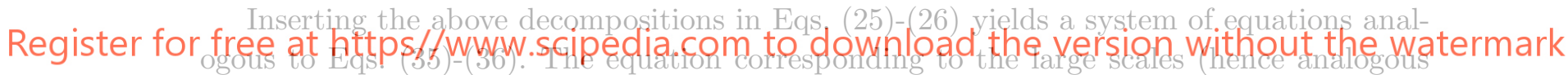
to Eq. (35)) becomes, after integrating some terms by parts and neglecting terms involving integrals over interelement boundaries ${ }^{17,18}$,

$$
\begin{aligned}
\left(\delta_{t} \boldsymbol{u}_{h}^{n}, \boldsymbol{v}_{h}\right)+ & \nu\left(\nabla \boldsymbol{u}_{h}^{n+1 / 2}, \nabla \boldsymbol{v}_{h}\right)+\left\langle\boldsymbol{u}_{h}^{n+1 / 2} \cdot \nabla \boldsymbol{u}_{h}^{n+1 / 2}, \boldsymbol{v}_{h}\right\rangle \\
& -\left(p_{h}^{n+1}, \nabla \cdot \boldsymbol{v}_{h}\right)+\left(q_{h}, \nabla \cdot \boldsymbol{u}_{h}^{n+1 / 2}\right) \\
& -\sum_{\Omega_{e l}}\left\langle\tilde{\boldsymbol{u}}^{n+1 / 2}, \nu \Delta \boldsymbol{v}_{h}+\boldsymbol{u}_{h}^{n+1 / 2} \cdot \nabla \boldsymbol{v}_{h}+\nabla q_{h}\right\rangle_{\Omega_{e l}} \\
& +\left(\delta_{t} \tilde{\boldsymbol{u}}^{n}, \boldsymbol{v}_{h}\right)+\left\langle\tilde{\boldsymbol{u}}^{n+1 / 2} \cdot \nabla \boldsymbol{u}_{h}^{n+1 / 2}, \boldsymbol{v}_{h}\right\rangle \\
& -\left\langle\tilde{\boldsymbol{u}}^{n+1 / 2}, \tilde{\boldsymbol{u}}^{n+1 / 2} \cdot \nabla \boldsymbol{v}_{h}\right\rangle \\
& -\left(\tilde{p}^{n+1}, \nabla \cdot \boldsymbol{v}_{h}\right)=l\left(\boldsymbol{v}_{h}\right) .
\end{aligned}
$$

The first two lines of Eq. (37) contain the Galerkin terms previously found in Eq. (29). The remaining lines correspond to stabilization terms that are by no means standard. The third line yields terms that are already obtained in the stabilization of the linearized and stationary version of the Navier-Stokes equations ${ }^{15,16}$ (Oseen problem). It is well-known that the inclusion of these terms in the formulation allows to circumvent the convection instabilities described in section 3.2.1, and to use equal interpolations for the velocity and 
pressure fields. The fourth and fifth lines contain terms arising from the effects of the velocity subscales, $\tilde{\boldsymbol{u}}$, in the material derivative of the equation. The first term in the fourth line accounts for the time derivative of the subscales, while the second term provides global momentum conservation ${ }^{18}$, which is not satisfied in the Galerkin finite element approach. The fifth line corresponds to a Reynolds stress for the subscales (note that $-\left\langle\tilde{\boldsymbol{u}}, \tilde{\boldsymbol{u}} \cdot \nabla \boldsymbol{v}_{h}\right\rangle=$ $\left.-\left\langle\tilde{\boldsymbol{u}} \otimes \tilde{\boldsymbol{u}}, \nabla \boldsymbol{v}_{h}\right\rangle\right)$. This term may be identified with the direct effects of the subscale turbulence onto the large scales, although the degree of its real influence on the results still needs further research ${ }^{18,40}$. Finally, the term in the sixth line accounts for the effects of the pressure subscales.

Our aim is to find now the solution $\boldsymbol{u}_{h}^{n+1 / 2}, \tilde{\boldsymbol{u}}^{n+1 / 2}$ and $p_{h}^{n+1}, \tilde{p}^{n+1}$ in Eq. (37), given $\boldsymbol{u}_{h}^{n}, \tilde{\boldsymbol{u}}^{n}$ and for all $\boldsymbol{v}_{h} \in \mathcal{V}_{0, h}^{d}, q_{h} \in \mathcal{Q}_{h, 0}$. Obviously, to do so we first need a value for the subscales $\tilde{\boldsymbol{u}}^{n+1 / 2}, \tilde{p}^{n+1}$ that has to be obtained from the solution of the small subgrid scales equation of the problem (analogous to Eq. (36)). This equation can be written in differential form as ${ }^{17,18}$

$$
\begin{aligned}
\delta_{t} \tilde{\boldsymbol{u}}^{n}+\left(\boldsymbol{u}_{h}^{n+1 / 2}+\tilde{\boldsymbol{u}}^{n+1 / 2}\right) \cdot \nabla \tilde{\boldsymbol{u}}^{n+1 / 2}-\nu \Delta \tilde{\boldsymbol{u}}^{n+1 / 2}+\nabla \tilde{p}^{n+1} & =\boldsymbol{r}_{u, h}^{n+1 / 2} \\
\nabla \cdot \tilde{\boldsymbol{u}}^{n+1 / 2} & =r_{p, h}^{n+1 / 2},
\end{aligned}
$$

with $\boldsymbol{r}_{u, h}^{n+1 / 2}$ and $r_{p, h}^{n+1 / 2}$ representing residuals of the finite element components $\boldsymbol{u}_{h}$ and $p_{h}$ given by

$$
\begin{aligned}
\boldsymbol{r}_{u, h}^{n+1 / 2} & =-\left[\delta_{t} \boldsymbol{u}_{h}^{n}+\left(\boldsymbol{u}_{h}^{n+1 / 2}+\tilde{\boldsymbol{u}}^{n+1 / 2}\right) \cdot \nabla \boldsymbol{u}_{h}^{n+1 / 2}\right. \\
& \left.-\nu \Delta \boldsymbol{u}_{h}^{n+1 / 2}+\nabla p_{h}-\boldsymbol{f}\right], \\
r_{p, h}^{n+1 / 2} & =-\left[\nabla \cdot \boldsymbol{u}_{h}^{n+1 / 2}\right] .
\end{aligned}
$$

Using arguments based on a Fourier analysis for the subscales ${ }^{17}$, the system of equations Eqs. (38)-(39) can be approximated as

$$
\begin{gathered}
\delta_{t} \tilde{\boldsymbol{u}}^{n}+\frac{1}{\tau_{1}^{n+1 / 2}} \tilde{\boldsymbol{u}}^{n+1 / 2}=\boldsymbol{r}_{u, h}^{n+1 / 2}, \\
\frac{1}{\tau_{2}^{n+1}} \tilde{p}^{n+1}=r_{p, h}^{n+1 / 2}
\end{gathered}
$$

where the so called stabilization parameters $\tau_{1}$ and $\tau_{2}$ have the expressions

$$
\begin{aligned}
\tau_{1}^{n+1 / 2} & =\left(c_{1} \frac{\nu}{h^{2}}+c_{2} \frac{\left|\boldsymbol{u}_{h}^{n+1 / 2}+\tilde{\boldsymbol{u}}^{n+1 / 2}\right|}{h}\right)^{-1}, \\
\tau_{2}^{n+1} & =\frac{h^{2}}{c_{1} \tau_{1}^{n+1}} .
\end{aligned}
$$

$c_{1}$ and $c_{2}$ in Eqs. (44)-(45) are algorithmic parameters with recommended values ${ }^{43}$ of $c_{1}=4$ and $c_{2}=2$, while $h$ stands for a characteristic element mesh size. From a physical point of 
view, the approximation Eqs. (42)-(43) to problem Eqs. (38)-(39) conserves the amount of kinetic energy transfered from the small scales to the large ones ${ }^{16}$.

Eq. (37) together with the subscales extracted from the solution of the approximated Eqs. (42)-(43) with stabilization parameters Eqs. (44)-(45) constitute the methodology herein proposed to solve the incompressible Navier-Stokes equations.

\subsection{Subgrid scale stabilized finite element method for the inhomogeneous Helmholtz equation}

We will now apply the subgrid scale approach to the Helmholtz equation weak form, Eq. (28). We will proceed as described by performing the space splitting $\mathcal{W}_{0}=\mathcal{W}_{0, h}+\tilde{\mathcal{W}}_{0}$, which allow the decompositions $\hat{p}^{\prime}=\hat{p}_{h}^{\prime}+\widetilde{\hat{p}}^{\prime}, w=w_{h}+\tilde{w}$ for the acoustic pressure and test function. Substitution in Eq. (28) yields the large scale and small scale equations. The former is given by ${ }^{44,1}$

$$
\begin{aligned}
\left(\nabla \hat{p}_{h}^{\prime}, \nabla w_{h}\right)-k^{2}\left(\hat{p}_{h}^{\prime}, w_{h}\right)-\mathrm{i} k\left(\hat{p}_{h}^{\prime}, w_{h}\right)_{\Gamma_{B}} & +\sum_{\Omega_{e l}}\left\langle\widetilde{\hat{p}}^{\prime}, \nabla^{2} w_{h}+k^{2} w_{h}\right\rangle_{\Omega_{e l}} \\
& =\left\langle\hat{s}_{h}, w_{h}\right\rangle+\left\langle\hat{g}, w_{h}\right\rangle_{\Gamma_{B}},
\end{aligned}
$$

where the first line contains the Galerkin terms already found in Eq. (32) and the second one accounts for the stabilization terms that take into account the influence of the small scales into the large ones. The small scales can be approximated as ${ }^{44,1}$

$$
\widetilde{\hat{p}^{\prime}}=\tau_{a c} r_{\hat{p}^{\prime}, h}=\tau_{a c}\left(-\nabla^{2} \hat{p}_{h}^{\prime}-k^{2} \hat{p}_{h}^{\prime}-\hat{s}_{h}\right) \text {. }
$$

The stabilization parameter $\tau_{a c}$ can be obtained from a dispersion analysis. The stencil of Eq. (46), with Eq. (47) inserted in it, is considered for a particular mesh, e.g. a structured mesh of bilinear quadrilateral nodes. Then a plane wave solution is assumed at each node and a dispersion relation is obtained from which a value for the stabilization parameter can be derived. This procedure was applied in Ref. 1 to find $\tau_{a c}$ for the convected Helmholtz equation in two dimensions. For the case of zero Mach number flow, the parameter thus obtained reduces to minus the one found in Ref. 42 using the Galerkin least-squares stabilized finite element method for the Helmholtz equation. $\tau_{a c}$ is given in this case by

$$
\begin{aligned}
\tau_{a c} & =-\frac{1}{k^{2}}+\frac{6}{k^{4} h^{2}} \frac{\left(4-f_{x}-f_{y}-2 f_{x} f_{y}\right)}{\left(2+f_{x}\right)\left(2+f_{y}\right)}, \\
f_{x} & =\cos [k \cos (\theta) h], \\
f_{y} & =\cos [k \sin (\theta) h],
\end{aligned}
$$

where $\theta$ is the angle of propagation of the plane wave and $h$ is the characteristic mesh element size. Even though the parameter $\tau_{a c}$ depends on the direction $\theta$ and on the particular mesh considered to derive it, numerical experiments show that the choice $\theta=0$ provides stabilization for a considerable variety of problems involving wave propagation in many directions. Moreover, Eq. (48) can be also shown to provide stabilization for non-structured meshes of quadrilateral and triangular elements ${ }^{1,21,23}$. 
Eq. (46), together with the subscales in Eq. (47) and the stabilization parameter from Eq. (48), clearly diminish the pollution error found in the Galerkin approximation to the problem and constitute the strategy adopted in this paper to compute the acoustic field. Obvioulsy, Eq. (48) limits the acoustic pressure computation to two-dimensional cases and a more general value for $\tau_{a c}$, or alternative stabilization strategies, should be used for full three dimensional problems ${ }^{22}$. However, it is to be noted that several acoustic problems can be treated, at least in a first approximation, as two dimensional problems. This is the case e.g., of duct acoustics, which may involve many different problems such as wave propagation in air conditioning systems, or models for voice generation mechanisms ${ }^{45}$.

\section{Numerical examples}

\subsection{Aeolian tone generated by a single cylinder at $\mathrm{Re}=500$}

We consider the case of a two-dimensional cylinder with diameter $D$ embedded in a flow with free stream velocity in Cartesian coordinates $\left(U_{0}, 0\right)$. We define the Reynolds number based on these variables as $\operatorname{Re}=\rho_{0} U_{0} D / \mu$. The following behavior occurs when $\operatorname{Re}$ is increased from an almost zero value to large values ${ }^{46,47}$ :

For $\operatorname{Re} \approx 0$ we have a Stokes flow and the configuration is totally symmetric: the flow is steady, time reversal and has up-and-down as well as fore-and-aft symmetries. When $\operatorname{Re} \approx 1$ the fore-and-aft symmetry breaks down and two steady recirculating vortices appear at the lee of the cylinder. These vortices grow in size for increasing Re. When $\operatorname{Re} \approx 45$ the flow becomes unstable and a Hopf bifurcation ${ }^{47}$ takes place. The flow looses its steadiness as well as its up-and-down symmetry and a wake of alternating vortices is formed behind the cylinder. The set of these shed vortices is known as the von Kármán vortex street. For a three-dimensional cylinder the story goes beyond as three-dimensional instabilities become excited for higher Reynolds numbers, until a fully developed turbulent flow is achieved probably following the Ruelle-Takens-Newhouse route to turbulence ${ }^{47}$.

Let us focus hereafter on the problem once the periodic von Kármán vortex street has been developed. As a reaction to vortex shedding, the cylinder supports lift fluctuations that lead to the emission of sound. The emitted noise is known as an aeolian tone and it is of concern in some industrial problems, such as noise radiated by train pantographs or by tubular heat exchangers. The mechanism is also responsible for the typical wire whistles that can be heard when wind impacts power transmission lines.

The aeolian tone has a dipole directivity and its frequency coincides with the vortex shedding frequency, which is given by

$$
f=\mathrm{S}_{\mathrm{t}} \frac{U_{0}}{D} .
$$

$\mathrm{S}_{\mathrm{t}}$ in Eq. (49) stands for the Strouhal number and is related to the Reynolds number by means of ${ }^{46}$

$$
\mathrm{S}_{\mathrm{t}}=0.198\left(1-\frac{19.7}{\operatorname{Re}}\right), \quad \operatorname{Re} \leq 5 \times 10^{5}
$$




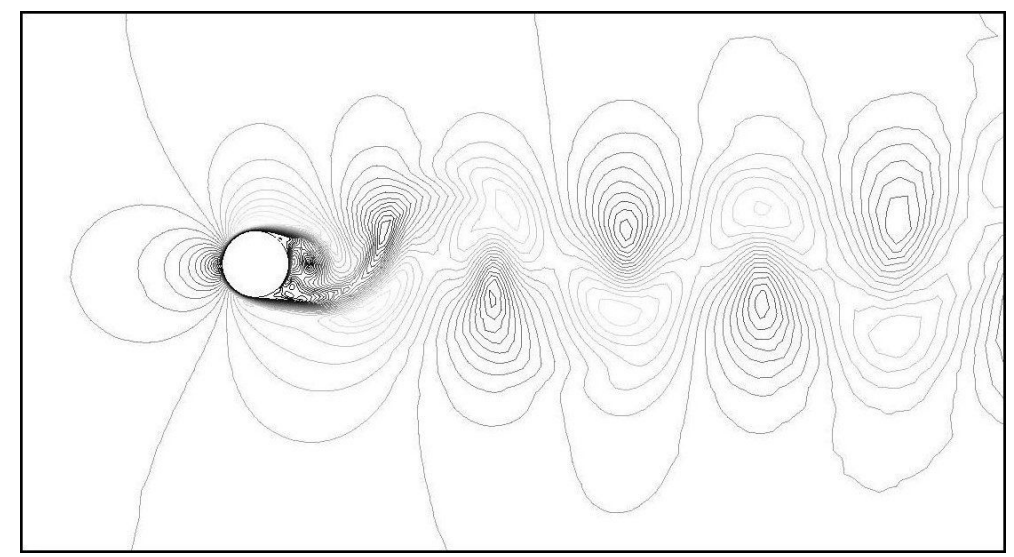

Fig. 1. Von Kármán vortex street at the lee of the cylinder for Re $=500$ (Isovelocity contourlines).
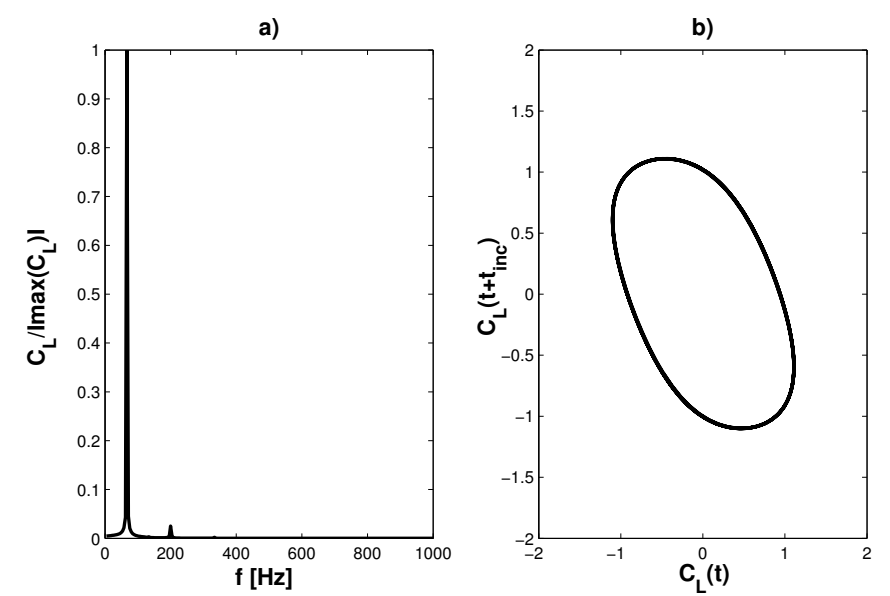

Fig. 2. $R e=500$. a) Lift coefficient spectrum. b) Phase space limit cycle.

For this first numerical example we have chosen a case corresponding to $R e=500$. The incompressible Navier-Stokes equations have been solved using the methodology described in section 4.2, in an unstructured mesh of linear triangular elements $\left(n_{\mathrm{el}}=50054, n_{\mathrm{u}}=\right.$ $n_{\mathrm{p}}=25636$ ). The mesh element size, $h$, ranges from $3 \times 10^{-3} D$ near the cylinder to $30 D$ at the far field. Equal interpolation has been used for the velocity and the pressure and 10 Picard non-linearity iterations have been performed at each time step. The time step size used in the computation is $\delta t=0.00025 \mathrm{~s}$.

The Strouhal number according to Eq. $(50)$ is $\mathrm{S}_{\mathrm{t}} \approx 0.19$. We have taken the values $D=0.0049 \mathrm{~m}$ and $U_{0}=1.512 \mathrm{~m} / \mathrm{s}$ so that the expected frequency from Eq. (49) is $f \approx 58.7 \mathrm{~Hz}$.

As a result of the simulation a periodic flow is established with a von Kármán vortex 


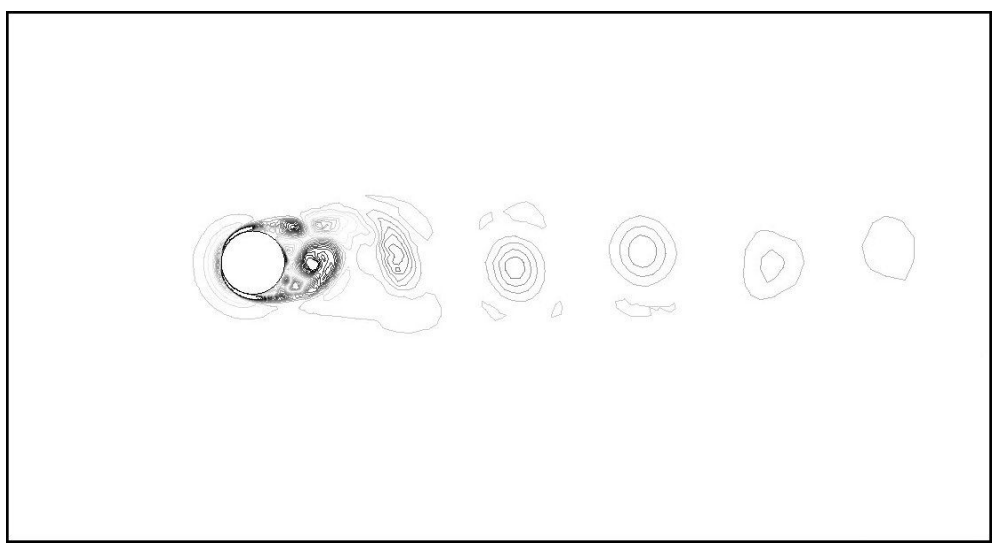

Fig. 3. Acoustic source term: snaphshot of Reynolds tensor double divergence for $R e=500$.

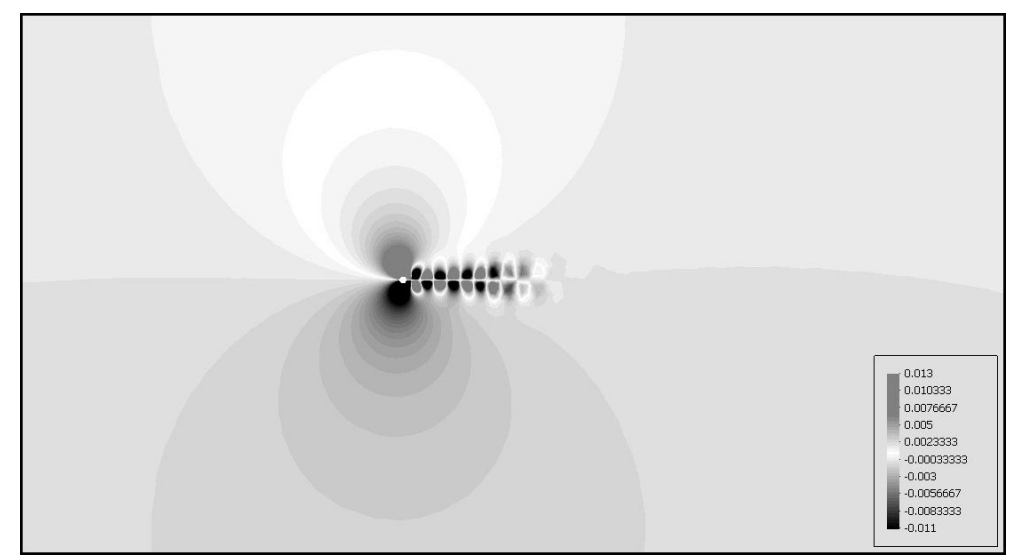

Fig. 4. Dipole pattern of $\operatorname{Im}\left(\hat{p}_{h}^{\prime}\right)$ at $f=66 \mathrm{~Hz}$ for $\mathrm{Re}=500$.

street developing at the lee of the cylinder (see Fig. 1). The lift and draft coefficients of the cylinder, $C_{L}$ and $C_{D}$, have been computed and present a time sinusoidal behavior. $C_{L}$ has an amplitude of 1.1 and oscillates at the computed vortex shedding frequency of $66 \mathrm{~Hz}\left(\mathrm{~S}_{\mathrm{t}}=0.21\right)$. The mean value for $C_{D}$ is 1.39 with an amplitude of 0.12 and a frequency that is twice the vortex shedding one $(132 \mathrm{~Hz})$. The discrepancy between the computed frequency and the theoretical one is not strange if we take into account that Eqs. (49)-(50) are valid for three dimensional flows and that $3 \mathrm{D}$ effects become apparent for $\mathrm{Re}>300$ (see Ref. 45).

In Fig. 2a we have plotted the normalized spectrum of the lift coefficient. As expected, it only shows a single peak at $66 \mathrm{~Hz}$. In Fig. $2 \mathrm{~b}$, we have presented a plot of $C_{L}(t)$ versus $C_{L}\left(t+t_{i n c}\right)$ with $t_{i n c}=0.05 \mathrm{~s}$. According to the Whitney-Takens theorem, the resulting graph is topologically equivalent to a phase space graph and we can observe that Fig. $2 \mathrm{~b}$ 
effectively shows the characteristic limit cycle of a periodic dynamics.

In Fig. 3 a snapshot of the acoustic source term $s(\boldsymbol{x}, t)=\rho_{0}(\nabla \otimes \boldsymbol{u}):(\nabla \otimes \boldsymbol{u})^{T}$ is shown. This term rapidly decreases to zero when moving away from the cylinder. This fact is of crucial importance because it actually justifies the acoustic analogy approaches, which are based on a separation between an acoustic source region and a wave propagating one $^{12,49}$.

The acoustic field has been computed according to the methodology described in section 4.3. In Fig. 4 the imaginary part of the acoustic pressure, $\operatorname{Im}\left(\hat{p}_{h}^{\prime}\right)$, is plotted. Although some acoustic sources can be identified at the wake of the cylinder (see Fig. 3), the far field acoustic field is clearly dominated by the lift fluctuations on the cylinder, which generate outward propagating waves having a clear dipole pattern.

\subsection{Aerodynamic noise generated by cylinders in parallel arrangement at $\mathrm{Re}=1000$}

As a second numerical example, we address the computation of the aerodynamic sound generated by a viscous flow impinging on two parallel cylinders with diameter $D$, and separated apart a distance of $3 D$ from center to center. The characteristic Reynolds number of the problem based on one cylinder's diameter and the impinging flow velocity is given by $\operatorname{Re}=\rho_{0} U_{0} D / \mu=1000$. We will show that vortices are periodically shed behind the two cylinders. However, due to the fact that these vortices become antiphase from one cylinder to the other, the resulting acoustic field will considerably differ from the single cylinder case.

The CFD simulation has been performed in a structured mesh of $n_{\mathrm{el}}=51485$ elements $\left(n_{\mathrm{u}}=n_{\mathrm{p}}=51881\right.$ nodes) strongly refined at the cylinder boundaries. The squared computational domain has a diagonal of $\sim 1000 D$. Again, the stabilised finite element formulation in section 4.2 has been used for the CFD calculation, with 10 Picard non-linearity iterations being performed at each time step. The time step size used in the computation is $\delta t=0.00008 \mathrm{~s}$. A second order Crank-Nicolson scheme has been used for the large scales time evolution, while a first order scheme has been used for the tracking of the subscales.

Once the initial transients have been surpassed, an almost periodic flow is established with vortices being shed past both cylinders. The vortices are antiphase from one to the other i.e., when a vortex having positive vorticity detaches from the upper cylinder, an equal strength vortex detaches from the bottom cylinder having negative vorticity, and being located at a symmetric location with respect to the $x$-axis. This can be clearly observed in Fig. 5 where the isovorticity contours of the flow have been plotted. It is worthwhile to comment that although the wake sometimes looses its symmetry downstream as time evolves, the vortex shedding remains periodic and antiphase between both cylinders.

In Fig. 6 we have plotted the time evolution for the lift coefficients $C_{L u}$ and $C_{L b}$ of the two cylinders. Once the flow is fully developed, their mean values are $\sim 0.19$ for the upper cylinder and $\sim-0.19$ for the one in the bottom. Their amplitudes are respectively \pm 1.6 . In what concerns the drag coefficients, they are obviously almost identical for both cylinders having a mean value of $\sim 1.53$ and an amplitude of $\sim 0.23$. In Figs. $7 \mathrm{a}$ and $\mathrm{b}$ we show the 


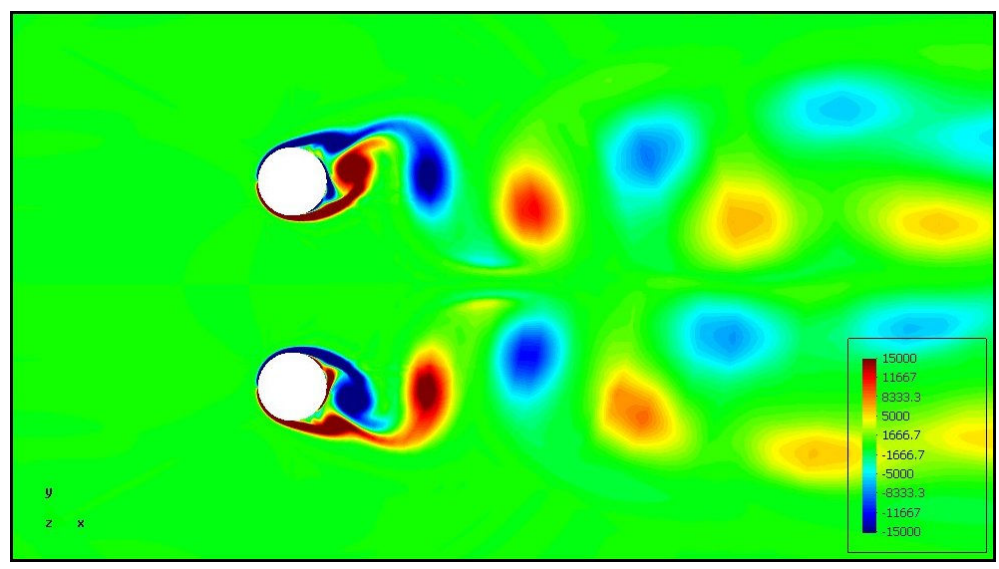

Fig. 5. Vortices shed behind two cylinders in parallel arrangement at Re $=1000$. (Isovorticity contours)

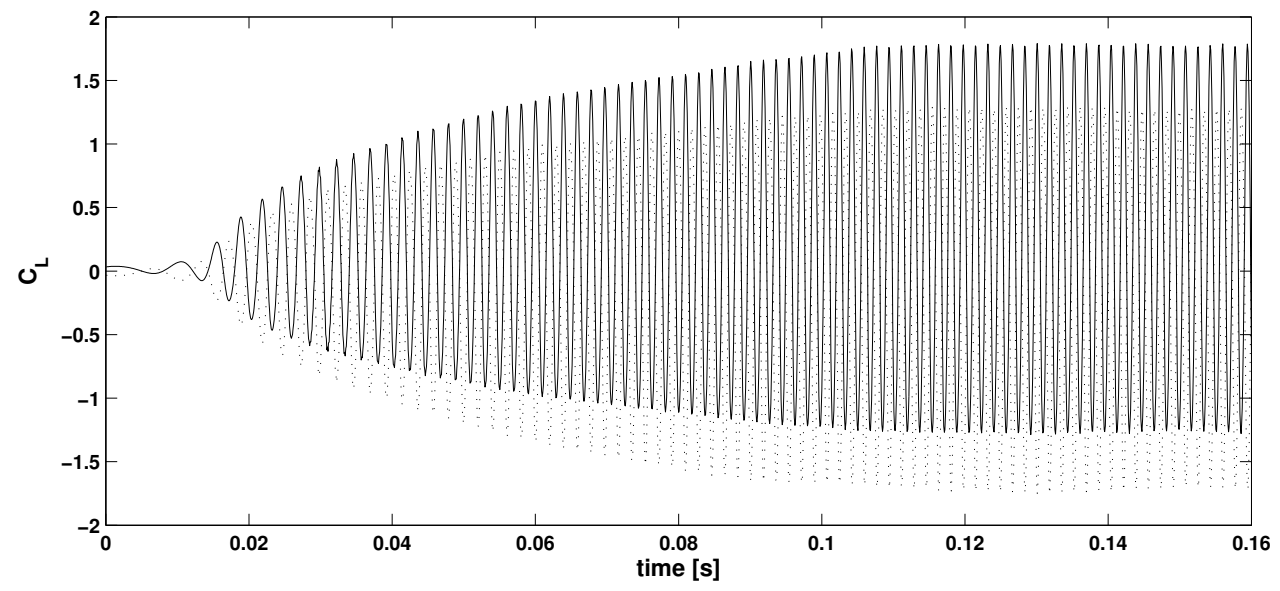

Fig. 6. Time evolution of the cylinder lift coefficients. Continuous line: upper cylinder. Dotted line: bottom cylinder. $\operatorname{Re}=1000$.

normalised spectra for the lift and drag coefficients of the upper cylinder. $C_{L u}$ presents a clear maximum at $588 \mathrm{~Hz}\left(\mathrm{~S}_{\mathrm{t}}=0.22\right)$ to be compared with the values $500 \mathrm{~Hz}\left(\mathrm{~S}_{\mathrm{t}}=0.19\right)$ arising from (49)-(50). The discrepancy has now increased when compared with the previous single cylinder numerical example probably for two reasons: first, the Reynolds number is now higher and second, equations (49)-(50) are intended for single cylinders whereas now the wake e.g., of the upper cylinder is clearly influenced by the wake of the bottom cylinder (see Fig. 5). We actually do not know if the presence of the second cylinder may alter the vortex shedding frequency when comparing with an analogous single cylinder case, but it certainly increases the complexity of the simulation. On the other hand, note that the normalised spectrum for the drag coefficient in Fig. $7 \mathrm{~b}$ presents the expected maximum 


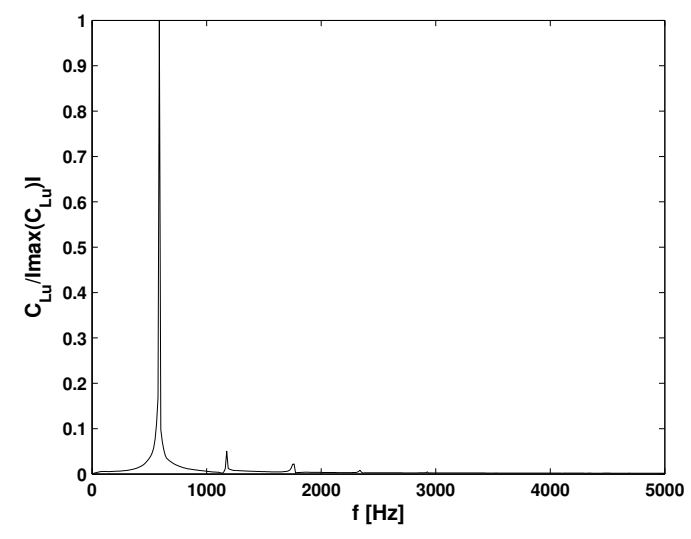

a) Lift coefficient

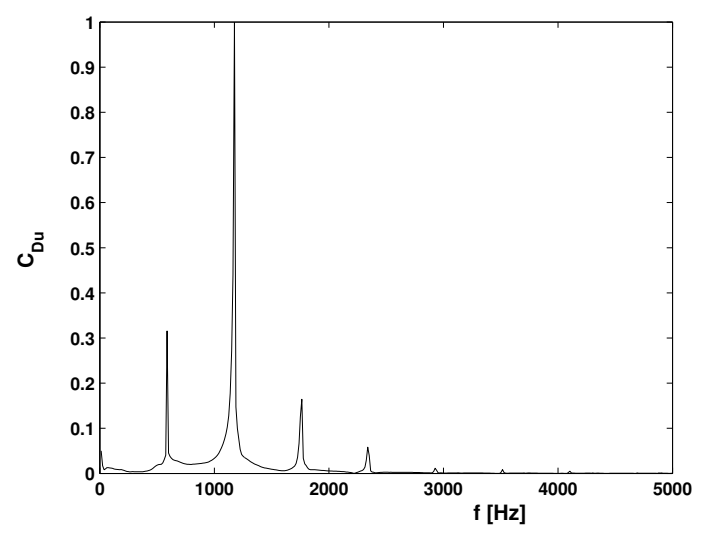

b) Drag coefficient

Fig. 7. Normalized lift and drag coefficient spectra for the upper cylinder. Re $=1000$.

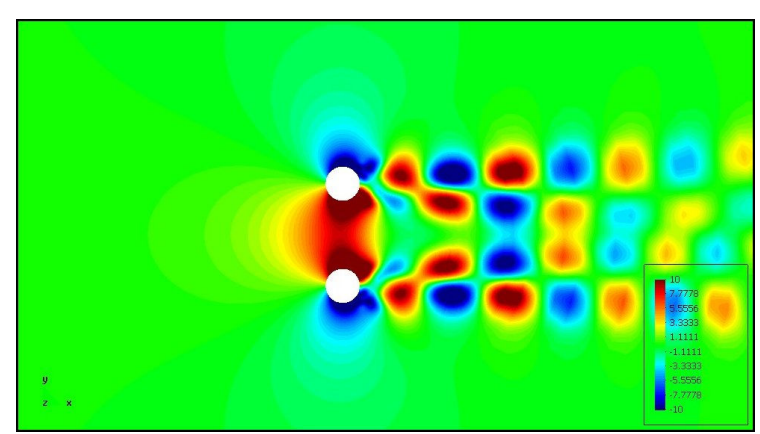

a) $\operatorname{Re}\left(\hat{p}_{h}^{\prime}\right)$

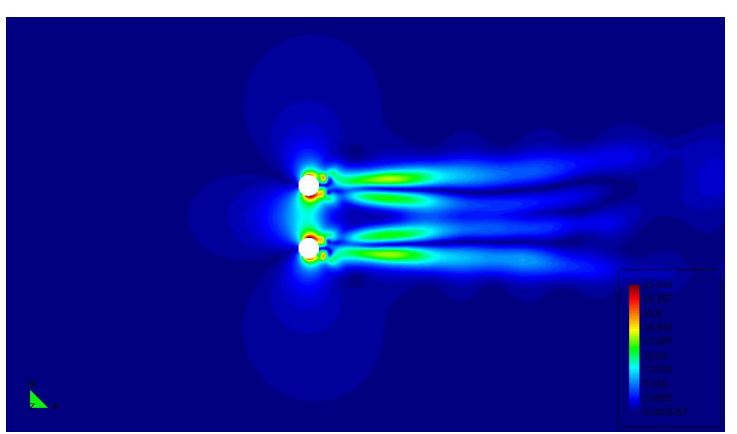

b) $\left|\hat{p}_{h}^{\prime}\right|$

Fig. 8. Acoustic near field. Cylinders in tandem arrangement at $\mathrm{Re}=1000$.

at $1176 \mathrm{~Hz}$ (twice the lift coefficient frequency) but it also presents a first subharmonic (lift coefficient frequency), a first harmonic and the first harmonic of fractional order. It is obvious that the drag coefficient is harder to compute given that it has twice the lift coefficient frequency. However, for the present simulation it still has more than ten time steps per wavelength. The appearance of the "extra" frequencies in Fig. $7 \mathrm{~b}$ is attributed to the fact that there is no perfect symmetry between the flow at the upper and bottom cylinders. In addition and as has been commented, the wake breaks downstream loosing also its symmetry. These facts can easily be responsible for the excitation of harmonics and subharmonics. Note that although the lift coefficient seems less affected by these questions, the second and third harmonics also insinuate in Fig. 7a.

In what concerns the acoustic field, it has again been computed using the procedure described in section 3.4.4. and using the same mesh intended for the CFD computation. In Figs. $8 \mathrm{a}$ and $\mathrm{b}$ we have plotted the real part of the near field acoustic pressure, $\operatorname{Re}\left(\hat{p}_{h}^{\prime}\right)$ 
and its modulus $\left|\hat{p}_{h}^{\prime}\right|$, for $f=588 \mathrm{~Hz}$. As seen in Fig. 8a, two dipole radiating patterns can be clearly identified at each cylinder corresponding to lift fluctuations. The two lift dipoles tend to compensate each other radiating as a single longitudinal quadrupole ${ }^{50}$ (see Fig. 8b), which is known to be a less efficient radiator than the dipole of a single cylinder. The quadrupole resemblance will be probably more apparent if the cylinders were closer one to each other. On the other hand, it has been checked that the wavelength of the generated acoustic waves have the expected value of $\lambda=c_{0} / f \sim 0.58 \mathrm{~m}$.

\section{Conclusions}

In this paper a methodology to compute aerodynamic noise generated by viscous subsonic flows has been presented. The approach makes use of Lighthill's acoustic analogy in the frequency domain. A three-step procedure has been followed consisting of a fluid dynamic computation of the incompressible Navier-Stokes equations to obtain the acoustic source term, its Fourier transform to the frequency domain and the computation of the acoustic pressure field.

The incompressible Navier-Stokes equation and the inhomogeneous Helmholtz equation appearing in the formulation have been solved by means of subgrid scale stabilized finite element methods. The subgrid scale stabilizing technique has allowed to circumvent several numerical problems appearing in the Galerkin finite element approach to these equations such as convection instabilities, the use of equal interpolation for the velocity and pressure fields, the use of small time steps or the appearance of the pollution error in the acoustic pressure field. The subgrid scale approach also provides an alternative way to simulate the behavior of turbulent flows, this point being a subject of intense current research.

The performance of the method when applied to computational aeroacoustics problems has been checked by simulating the aerodynamic noise generated by flow past a single two dimensional cylinder and by flow past two cylinders in tandem arrangement. The flow dynamics and the directivity character of the acoustic field have been correctly reproduced.

\section{Acknowledgments}

This research has been partially funded by the research project DPI2000-0431-P4-03 of the Ministerio de Ciencia y Tecnología and by the CIDEM research project RDITCRD05-10010 of the Generalitat de Catalunya. On the other hand, this work was mainly carried out while the first author was working at ICR (Engineering for the Noise Control) and at CIMNE (International Center for Numerical Methods in Engineering). Both entities are acknowledged for their support.

\section{References}

1. O. Guasch and R. Codina, An algebraic subgrid scale finite element method for the convected Helmholtz equation in two dimensions with applications in aeroacoustics, Comput. Methods Appl. Mech. Engrg. 196 (45-48), 4672-4689 (2007). 
2. M. Lighthill, On sound generated aerodinamically I. General theory, Proc. R. Soc. Lond. 211 A 1107, 564-587 (1952).

3. N. Curle, The influence of solid boundaries on aerodynamic sound, Proc. R. Soc. Lond. 231 A 1187, 505-514 (1955).

4. J. Ffowcs-Williams and D. Hawkings, Sound generated by turbulence and surfaces in arbitrary motion, Phil. Trans. Roy. Soc. A 264, 321-342 (1969).

5. J. Ffowcs-Williams, Hydrodynamic noise, Annu. Rev. Fluid Mech. 1, 197-222 (1969).

6. M. Goldstein, Unified approach to aerodynamic sound generation in the presence of solid boundaries, J. Acoust. Soc. Am. 56, 497-509 (1974).

7. G. Lilley, On the noise radiated from a turbulent high speed jet, in Computational Aeroacoustics, J.C. Hardin and M.Y. Hussaini (Eds.) (Springer Verlag) (1993).

8. A. Powell,Theory of vortex sound, J. Acoust. Soc. Am. 36 (1964).

9. M. Howe, The generation of sound by aerodynamic sources in an inhomogeneous steady flow, $J$. Fluid Mech. 67 (3), 597-610 (1975).

10. W. Möhring, On vortex sound at low Mach number, J. Fluid Mech. 85, 685-691 (1978).

11. M. Goldstein,A generalized acoustic analogy, J. Fluid Mech. 488 (3), 315-333 (2003).

12. S. Crow, Aerodynamic sound emission as a singular perturbation problem, Stud. Appl. Math. 49 (1), 21-44 (1970).

13. J. Ristorcelli, A closure for the compressibility of the source terms in Lighthill's acoustic analogy, Technical Report ICASE Report 97-44, NASA/CR-1997-201738 (1997).

14. S. Slimon, M. Soteriou, and D. Davis, Development of computational aeroacoustics equations for subsonic flows using a Mach number expansion approach, J. Comput. Phys. 159, 377-406 (2000).

15. R. Codina, Stabilization of incompressibility and convection through orthogonal sub-scales in finite element methods, Comput. Methods Appl. Mech. Engrg. 190, 1579-1599 (2000).

16. R. Codina, A stabilized finite element method for generalized stationary incompressible flows, Comput. Methods Appl. Mech. Engrg. 190, 2681-2706 (2001).

17. R. Codina, Stabilized finite element approximation of transient incompressible flows using orthogonal subscales, Comput. Methods Appl. Mech. Engrg. 191, 4295-4321 (2002).

18. R. Codina, J. Principe, O. Guasch, and S. Badia, Time dependent subscales in the stabilized finite element approximation of incompressible flow problems, Comput. Methods Appl. Mech. Engrg. 196 (21-24), 2413-2430 (2007).

19. J. Principe, R. Codina, and F. Henke, The dissipative structure of variational multiscale methods for incompressible flows, Comput. Methods Appl. Mech. Engrg. In press (2008).

20. O. Guasch and R. Codina, A heuristic argument for the sole use of numerical stabilization with no physical LES modeling in the simulation of incompressible turbulent flows, Submitted (2008).

21. L. Thompson and P. Pinsky, A Galerkin least-squares finite element method for the twodimensional Helmholtz equation, Int. J. Numer. Meth. Engrg. 38, 371-397 (1995).

22. I. Harari A survey of finite element methods for time harmonic acoustics, Comput. Methods Appl. Mech. Engrg. 195, 1594-1607 (2006).

23. I. Harari and F. Magoulès Numerical investigations of stabilized finite element computations for acoustics, Wave Motion 39, 339-349 (2004).

24. A. Oberai, F. Roknaldin, and T. Hughes, Computational procedures for determining structuralresponse due to hydrodynamic sources, Comput. Methods Appl. Mech. Engrg. 190, 345-361 (2000).

25. A. Oberai, F. Roknaldin, and T. Hughes, Computation of trailing-edge noise due to turbulent flow over an airfoil, AIAA J. 40(11), 2206-2216 (2002).

26. F. Bastin, P. Lafon, and S. Candel, Computation of jet mixing noise due to coherent structures: the plane jet case, J. Fluid Mech. 335, 261-304 (1997). 
27. P. Lockard, An efficient, two-dimensional implementation of the Ffwocs-Williams and Hawkings equation, J. Sound Vibr. 229, 897-911 (2000).

28. F. Farassat, The acoustic analogy as a tool of computational aeroacoustics, in Computational Aeroacoustics, edited by J. Hardin and M. Hussaini (Springer-Verlag) (1993).

29. C. Tam and J. Webb, Dispersion-relation-preserving schemes for computational acoustics, J. Comput. Phys. 107, 262-281 (1993).

30. C. Cheong and S. Lee, Grid-optimized dispersion-relation-preserving schemes on general geometries for computational aeroacoustics, J. Comput. Phys. 174, 248-276 (2001).

31. V. Wells and R. Renaut, Computing aerodynamically generated noise, Annu. Rev. Fluid Mech. 29, 161-199 (1997).

32. S. Chang, X. Wang, and C. Chow, The space-time Conservation-Element and Solution-Element method. a new high resolution and genuinely multidimensional paradigm for solving conservation laws, J. Comput. Phys. 159, 89-136 (1999).

33. S. Pope, Turbulent Flows (Cambridge University Press) (2000).

34. P. Sagaut, Large Eddy Simulation for Incompressible Flows (Scientific Computing, Springer) (2001).

35. T. Hughes, Multiscale phenomena: Green's function, the Dirichlet-to-Neumann formulation, subgrid scale models, bubbles and the origins of stabilized formulations, Comput. Methods Appl. Mech. Engrg. 127, 387-401 (1995).

36. T. Hughes, G. Feijóo, L. Mazzei, and J. Quincy, The variational multiscale method-a paradigm for computational mechanics, Comput. Methods Appl. Mech. Engrg. 166, 3-24 (1998).

37. T. Hughes, L. Mazzei, and K. Jansen, Large eddy simulation and the variational multiscale method, Comput. Visual. Sci. 3, 47-59 (2000).

38. T. Hughes, L. Mazzei, and A. Oberai, The multiscale formulation of large eddy simulation: Decay of homogeneous isotropic turbulence, Phys. Fluids 13(2), 505-512 (2001).

39. T. Hughes, L. Mazzei, and A. Oberai, Large eddy simulation of turbulent channel flow by the variational multiscale method, Phys. Fluids 13(6), 1784-1798 (2001).

40. V. Calo, Residual based multiscale turbulence modeling: finite volume simulations of bypass transition, PhD. thesis, Stanford University (2004).

41. Y. Bazilevs, V. Calo, J. Cottrell, T. Hughes, A. Reali, and G. Scovazzi, Variational multiscale residual-based turbulence modeling for large eddy simulation, Comput. Methods Appl. Mech. Engrg. 197 (1-4), 173-201 (2007).

42. F. Ihlenburg, Finite Element Analysis of Acoustic Scattering (Applied Mathematical Sciences 132, Springer Verlag) (1998).

43. R. Codina, On stabilized finite element methods for linear systems of convection-diffusionreaction equations, Comput. Methods Appl. Mech. Engrg. 188, 61-82 (2000).

44. R. Codina, Comparison of some finite element methods for solving the diffusion-convection-reaction equation, Comput. Methods Appl. Mech. Engrg. 156, 185-210 (1998).

45. T. Kako and K. Touda, Numerical method for voice generation problem based on finite element method, J. Comput. Acoust. 14(1), 45-56 (2006).

46. M. S. Howe, Acoustics of Fluid-Structure Interactions (Cambridge University Press) (1998).

47. P. Drazin, Introduction to Hydrodynamic Stability (Cambridge Texts in Applied Mathematics. Cambridge University Press) (2002).

48. S. Mittal and V. Kumar, Flow-induced vibrations of a light circular cylinder at Reynolds numbers $10^{3}$ to $10^{4}$, J. Sound Vibr. 245(5), 923-946 (2001).

49. D. Crighton, Computational aeroacoustics for low Mach number flows, in Computational Aeroacoustics, edited by J. Hardin and M. Hussaini (Springer-Verlag) (1993).

50. D. Russell, On the sound field radiated by a tuning fork, Am. J. Phys. 68(12), 1139-1145 (2001). 